\title{
Local Cerebral Glucose Utilization in Monkeys with Hemiparkinsonism Induced by Intracarotid Infusion of the Neurotoxin MPTP
}

\author{
Ernesta Palombo, ${ }^{1}$ Linda J. Porrino, ${ }^{1,2}$ Krystof S. Bankiewicz, ${ }^{3}$ Alison M. Crane, ${ }^{1}$ Louis Sokoloff, ${ }^{1}$ and Irwin J. \\ Kopin ${ }^{2}$ \\ 'Laboratory of Cerebral Metabolism, National Institute on Mental Health, Bethesda, Maryland 20892, ${ }^{2}$ Clinical \\ Neuroscience Branch and ${ }^{3}$ Surgical Neurology Branch, National Institute of Neurological Disorders and Stroke, Bethesda, \\ Maryland 20892
}

\begin{abstract}
Quantitative 2-[ $\left.{ }^{14} \mathrm{C}\right]$ deoxyglucose autoradiography was used to map the pattern of alterations in local cerebral glucose utilization associated with unilateral lesions of the substantia nigra pars compacta produced by the infusion of 1-methyl4-phenyl-1,2,3,6-tetrahydropyridine (MPTP) into one internal carotid artery of rhesus monkeys. These monkeys become hemiparkinsonian, displaying rigidity, bradykinesia, and tremor of the limbs contralateral to the side of MPTP infusion; during spontaneous activity they turn toward the side of the lesion. Eighty-two brain areas were examined, and statistically significant metabolic changes were confined mainly to basal ganglia structures ipsilateral to the side of the lesion. Glucose utilization was reduced in the substantia nigra pars compacta and ventral tegmental area, i.e., in the areas of cell loss. Increases in glucose utilization in regions normally innervated by the lesioned area were observed in the postcommissural portions of the putamen and dorsolateral caudate. Other structures showing statistically significant metabolic changes were the external segment of the globus pallidus $(+40 \%)$, subthalamic nucleus $(-17 \%)$, and pedunculopontine nucleus $(+15 \%)$. There were also smaller changes in portions of the thalamus (ventral anterior nucleus, parafascicular nucleus) and premotor cortex. All significant metabolic changes were confined to the side of the substantia nigra lesion and were essentially restricted to regions involved in the production of movement or maintenance of posture.
\end{abstract}

The discovery that systemic administration of the neurotoxin 1-methyl-4-phenyl-1,2,3,6-tetrahydropyridine (MPTP) destroys dopaminergic neurons of the substantia nigra pars compacta (SNpc) and induces parkinsonism in humans (Langston et al., 1983) and subhuman primates (Burns ct al., 1983) has provided a valuable tool to investigate the effects of nigrostriatal denervation and its relationship to parkinsonian symptoms. Subhuman primates treated systematically with MPTP develop

\footnotetext{
Received July 12, 1989; revised Sept. 13, 1989; accepted Sept. 15, 1989.

We thank Jane Jehle for her excellent technical assistance and Dory Feldman for her help in the preparation of this manuscript.

Correspondence should be addressed to Linda J. Porrino, Ph.D., Clinical Neuroscience Branch, NINDS, 9000 Rockville Pike, Building 10, Room 5N-214, Bethesda, MD 20892.

0270-6474/90/030860-09\$02.00/0
}

a bilaterally symmetrical parkinsonian syndrome of postural rigidity, bradykinesia, akinesia, tremor, and loss of facial expression.

The infusion of MPTP into one internal carotid artery of primates damages dopaminergic cells of the SNpc ipsilateral to the side of the infusion with little or no apparent damage to the contralateral side, thus producing a unilateral parkinsonian or hemiparkinsonian syndrome (Bankiewicz et al., 1986). While this model has many similarities to the model of parkinsonism in rodents produced by the unilateral intracerebral injection of 6-hydroxydopamine (Ungerstedt, 1971), intracarotid MPTP lesions are more specific in that there is relative sparing of dopamine-containing neurons outside the SNpc (Bankiewicz et al., 1986). Hemiparkinsonian monkeys display bradykinesia, rigidity, and tremor in the limbs contralateral to the side of the lesion and spontaneous turning toward the side of the lesion. Although there have been reports of behavioral recovery in some species of monkeys treated unilaterally with MPTP (Brewer et al., 1988), we have not observed spontaneous recovery. The parkinsonian signs and symptoms remain stable for as long as a year after MPTP treatment.

Dopamine levels in the caudate and putamen on the MPTPtreated side of hemiparkinsonian monkeys are decreased by more than $95 \%$ when compared to the nontreated side or to control monkeys (Bankiewicz et al., 1986; Kopin et al., 1988), and striatal dopamine and tyrosine hydroxylase levels on the nontreated side do not differ significantly from those of control animals (Kopin et al., 1988). The number of tyrosine hydroxylase-like immunoreactive cells present in the SNpc on the MPTP-treated side is also reduced by more than $90 \%$, and the number of cells on the nontreated side has been found to be within the range of the number of cells in normal control monkeys (Cummins et al., 1989).

Because of the close relationship between rates of energy metabolism and local functional activity, the $2-\left[{ }^{14} \mathrm{C}\right]$ deoxyglucose (2DG) method (Sokoloff et al., 1977) has been used by various investigators to identify the underlying neural substrates involved in the development and manifestation of the signs and symptoms of MPTP-induced parkinsonism (Schwartzman and Alexander, 1985; Mitchell et al., 1986; Porrino et al., 1987a, b; Ho et al., 1988; Palombo et al., 1988; Schwartzman et al., 1988). In the present study the 2DG method (Sokoloff et al., 1977) was used to define the regional distribution of cerebral metabolic 
changes associated with hemiparkinsonism and to compare them with those previously found to accompany full chronic bilateral parkinsonism.

\section{Materials and Methods}

Animals. Eight Macaca mulatta monkeys of both sexes (6 females; 2 males) were used. The monkeys were housed in quarters with a $12-\mathrm{hr}$ light/dark cycle and fed Purina monkey chow twice daily with water available ad libitum. Four of these monkeys were made hemiparkinsonian by unilateral intracarotid infusion of MPTP; 4 monkeys were used as normal controls.

Unilateral lesion of the SNpc. Monkeys were anesthetized with ketamine and xylazine i.m. ( 7 and $0.6 \mathrm{mg} / \mathrm{kg}$, respectively); a catheter was inserted via the right femoral artery into the right or left carotid artery under fluoroscopic control. MPTP solution $(0.03 \mathrm{mg} / \mathrm{ml}$ of saline) was infused at a rate of $3.8 \mathrm{ml} / \mathrm{min}$ (total dosc $2.5 \mathrm{mg}$ in $80 \mathrm{ml}$ of salinc) and the monkeys returned to their cages. This infusion schedule was chosen to minimize exposure of the contralateral hemisphere to MPTP (Bankiewicz et al., 1986). Clinical and behavioral signs (tremor, rigidity, limb use, and oculomotor function) were (tremor, rigidity, limb use, and oculomotor function) were assessed daily for the first several weeks after MPTP treatment and weekly thereafter. In addition, contralateral turning was evaluated after apomorphine $(0.2 \mathrm{mg} / \mathrm{kg}$, i.m. $)$ administration; each animal was tested 3 or 4 times. An initial test was made during the first several weeks following MPTP treatment, and a final test was performed between 5 and $14 \mathrm{~d}$ prior to the $2 \mathrm{DG}$ procedure. Hemiparkinsonian monkeys did not require, nor did they receive, any pharmacological therapy.

Physiological variables. Physiological variables (mean arterial blood pressurc, hematocrit, $\mathrm{pH}, \mathrm{pO}_{2}, \mathrm{pCO}_{2}$, plasma glucose concentration) were measured $30 \mathrm{~min}$ before the beginning of the $2 \mathrm{DG}$ procedure. Mean arterial blood pressure was measured with an air-damped mercury manometer. Arterial gases and $\mathrm{pH}$ were measured with a Model 158 pH/Blood Gas Analyzer (Corning Medical, Medfield, MA). Glucose concentrations in plasma were measured with a Beckman Glucose Analyzer II (Beckman Instruments, Fullerton, CA).

Measurement of local cerebral glucose utilization. Local cerebral glucose utilization (LCGU) was measured by means of the 2DG method (Sokoloff et al., 1977; Kennedy et al., 1978) 1.5-5 months after MPTP infusion. The monkeys were lightly anesthetized with a mixture of halothane and nitrous oxide, and polyethylene catheters were inserted into a femoral artery and vein for subsequent intravenous administration of 2DG and sampling of arterial blood. Animals were then seated in a primate chair and allowed to recover from the ancsthesia and surgery for a minimum of $3 \mathrm{~h}$. The experimental period was initiated by injection of an intravenous pulse of $100 \mu \mathrm{Ci} / \mathrm{kg}$ of 2-deoxy-D-[1-14 C]glucose (specific activity $50-55 \mathrm{mCi} / \mathrm{mmol}$, New England Nuclear, Boston, MA). Timed arterial blood samples were drawn thereafter at a schedule sufficient to define the time course of the arterial $2-\left[{ }^{14} \mathrm{C}\right] \mathrm{DG}$ concentration. Blood samples were centrifuged immediately; plasma concentrations of $2-\left[{ }^{14} \mathrm{C}\right] \mathrm{DG}$ were determined by liquid scintillation spectrometry (Beckman Instruments, Model LS-255, Fullerton, CA), and plasma glucose concentrations measured as described above. After approximately 45 min the animals were killed by an intravenous overdose of sodium pentobarbital. The brains were rapidly removed, blocked in 3 parts, frozen in isopentane $\left(-45^{\circ} \mathrm{C}\right)$, and stored at $-70^{\circ} \mathrm{C}$ until processed for autoradiography. Four $20-\mu \mathrm{m}$-thick coronal sections of brain every 500 $\mu \mathrm{m}$ were cut in a cryostat maintained at $-22^{\circ} \mathrm{C}$, thaw-mounted on glass coverslips, dried on a hot plate, and autoradiographed with Kodak OM1 $\mathrm{X}$-ray films, along with a set of $\left[{ }^{14} \mathrm{C}\right]$ methylmethacrylate standards (Amersham, Arlington Heights, IL) that had been previously calibrated for their equivalent ${ }^{14} \mathrm{C}$ concentrations in $20-\mu \mathrm{m}$ brain sections (Reivich et al., 1969). Autoradiograms were analyzed by quantitative densitometry with a computer-assisted image processing system (Goochee et al., 1980). Optical density measurements for each structure were made for each side in a minimum of 8 brain sections. Measurements of each structure were made in each section in which the structure was visible. In each case as large an area as possible was included in the measurement. Tissue ${ }^{14} \mathrm{C}$ concentrations were determined from the ontical densities and a calibration curve obtained by densitometric analysis of the autoradiograms of the calibrated standards. Local glucose utilization was then calculated from the local tissue ${ }^{14} \mathrm{C}$ concentrations, the time course of the plasma $\left[{ }^{14} \mathrm{C}\right] \mathrm{DG}$ and glucose concentrations, and the ap- propriate kinetic constants for the monkey (Kennedy et al., 1978) by the operational equation of the 2DG method (Sokoloff et al., 1977).

Statistical analysis. Physiological variables in the control and hemiparkinsonian groups of animals were compared by means of the twotailed Student $t$ test. Statistical analysis was carried out separately on LCGU values obtained for each individual cerebral structure, by means of a 2-way analysis of variance (ANOVA) for repeated measures with MPTP treatment as the independent factor and side of the brain as the repeated measure. This was followed by Bonferroni $t$ tests for multiple comparisons. Comparisons were made between (1) values in the sides ipsilateral and contralateral to the lesion in the hemiparkinsonian monkeys; (2) values in the side of the lesion in hemiparkinsonian animals with the corresponding side in the controls; and (3) values on the side contralateral to the lesion in hemiparkinsonian animals with the corresponding side of control animals. This relatively conservative statistical approach was utilized in order to decrease the probability of spurious statistically significant findings because of the large number of total comparisons made.

\section{Results}

\section{Physiological variables}

No statistically significant differences were found between control animals and MPTP-treated animals for the variables examined, i.e., plasma glucose, arterial blood pressure, hematocrit, and arterial $\mathrm{pCO}_{2}, \mathrm{pO}_{2}$, and $\mathrm{pH}$ (data not shown).

\section{Clinical signs and behavior}

Unilateral infusions of MPTP resulted in contralateral hemiparkinsonian signs, such as bradykinesia, rigidity, and occasional postural tremor. The forelimb ipsilateral to the lesion was preferentially used by the hemiparkinsonian monkeys. Marked tonic deviation of the head and gaze toward the side of the lesion, as well as spontaneous turning ipsilateral to the MPTP lesion (up to 60 rotations in $1 \mathrm{hr}$ ) were also observed. The clinical signs of hemiparkinsonism appeared in the first week after the infusion of MPTP, followed during the next 2-3 weeks by a transient dystonic posture of the hindlimb contralateral to the MPTP-infused side. By 5 weeks dystonic postures had disappcared, and hemiparkinsonian signs had become stable. At the time of the 2DG experiments (1.5-5.7 months postinfusion) no further clinical progression or evidence of recovery was seen.

All animals displayed rotation contralateral to the side of the lesion in response to apomorphine challenge. The degree of turning behavior varied among animals $(250 \pm 84$, mean \pm $\mathrm{SD}$; range 47-527 rotations/hr), but the behavior of each individual hemiparkinsonian monkey was relatively constant across tests over time (within $20 \%$ of the average).

\section{Histology}

Neuronal cell loss in the SNpc on the side infused with MPTP was evident in thionin-stained sections, and there was no overt evidence of gross histological abnormalities in the SNpc on the nonlesioned side or in other brain areas. Formal cell counts were not performed in these animals, because fresh frozen tissue necessary for autoradiography is not of sufficient quality to be processed appropriately for reliable cell counting. Cell counts, however, in animals similarly treated have revealed cell losses of greater than $90 \%$ within the SNpc on the MPTP-treated side and normal cell densities on the nonlesioned side (Cummins et al., 1989). One hemiparkinsonian monkey had a relatively large cystic lesion in the temporal pole and a small lesion in the rostral tip of the internal segment of the globus pallidus on the side of the SNpc lesion. Because the lesions were old, and presumably preexistent to the MPTP treatment, and because the behavior and changes of glucose utilization in this monkey did not differ 
Figure 1. Autoradiogram of coronal section of brain at the level of the mesencephalic-diencephalic junction. Optical densities are proportional to $\left[{ }^{14} \mathrm{C}\right]-$ deoxyglucose uptake, which reflects rates of glucose utilization (see tables for corresponding quantitative data). The substantia nigra pars compacta (SNpc) shows a marked decrease in deoxyglucose uptake on the lesioned side, where cell loss occurs (Bankiewicz et al., 1986). No asymmetries in density

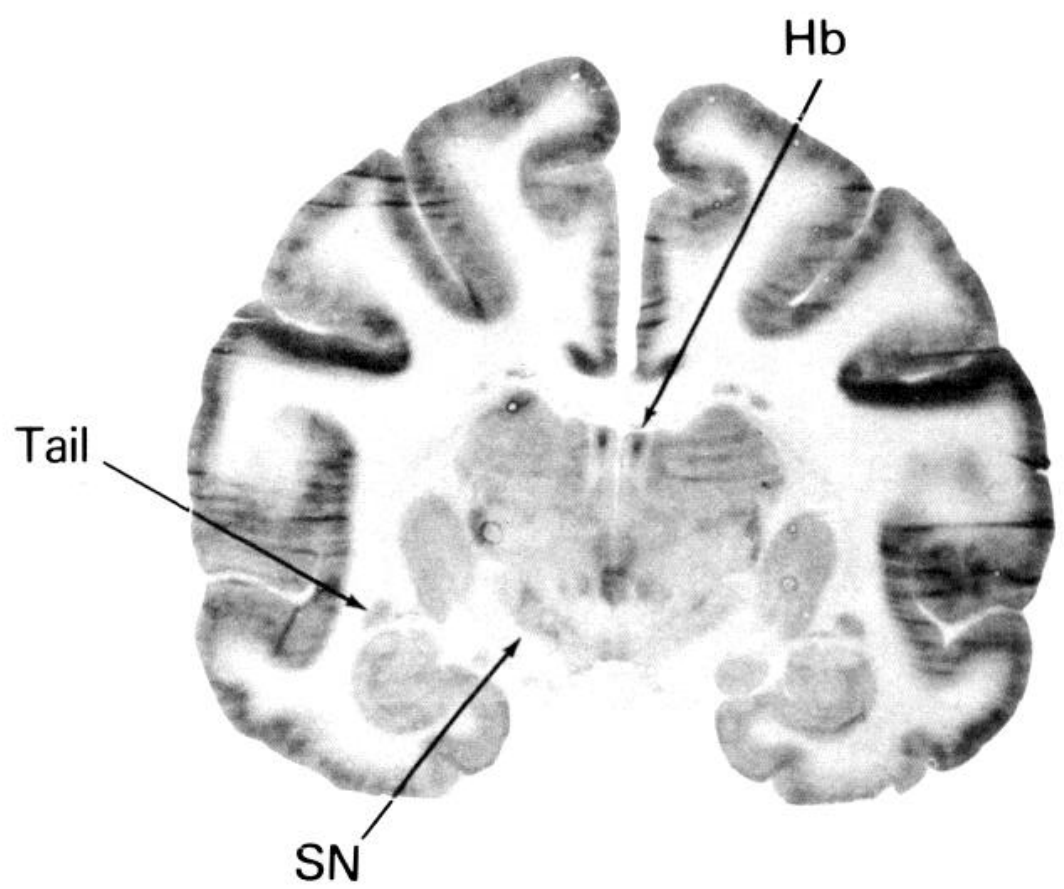
are evident in other regions.

\section{MPTP-Lesioned Side}

from those of the other hemiparkinsonian monkeys, it was included in this study.

\section{Glucose utilization}

Statistically significant changes in rates of glucose utilization were found in 14 of the 82 brain regions examined (Tables 15 ) in monkeys with MPTP-induced lesions of the SNpc. Most of the affected structures were either within the basal ganglia or

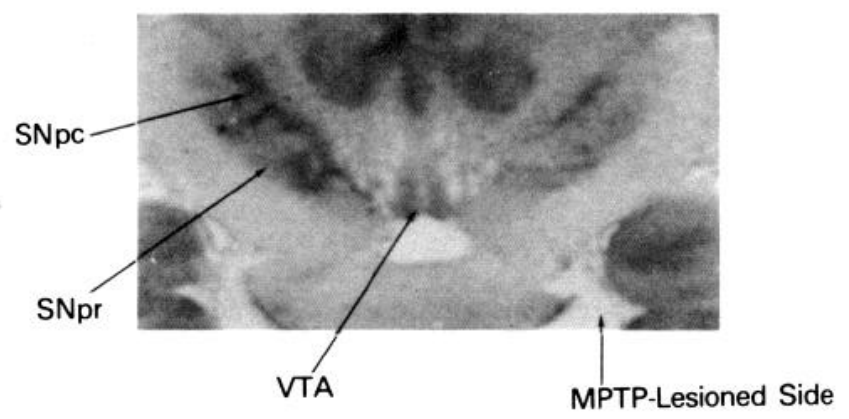

Figure 2. Autoradiogram of coronal section at the level of the substantia nigra showing greater detail than Figure 1. A difference in labeling of the substantia nigra pars compacta $(S N p c)$ ipsilateral and contralateral to the side of the lesion is evident. Furthermore, on the side of the lesion, label is unevenly distributed within the SNpc: The most medial band and all ventral extensions of the SNpc show the most marked reductions of deoxyglucose uptake with sparing of the dorsolateral portions. The metabolic activity of the substantia nigra pars reticulata ( $S N p r$ ) is unchanged, as seen by symmetrical distribution of the label in the area above the cerebral peduncle intermingled with the ventral extensions of the $\mathrm{SNpc}$. At this level no asymmetry is readily evident in the ventral tegmental area $(V T A)$ although full quantification of glucose utilization shows a significant decrement in metabolic rates on the side infused with MPTP (Table 2). in structures anatomically and functionally related to them. Rates of glucose utilization were decreased in the $\mathrm{SNpc}$ on the side of the lesion (Table 1; Figs. 1, 2); the decreases were most prominent in the medial and central portions of the SNpc (Fig. 2). In the mesencephalic tegmentum, the ventral tegmental area (VTA), nucleus paranigralis, and nucleus parabrachialis pigmentosus also showed significantly reduced rates of glucose utilization following MPTP administration (Table 1). Significant reductions of glucose utilization in both the $\mathrm{SNpc}$ and ventral tegmentum were limited only to the side ipsilateral to the MPTP infusion, except for the nucleus parabrachialis pigmentosus (Table 1), where the reductions were bilateral as compared to controls.

Within the basal ganglia, glucose utilization was significantly increased in the external segment of the globus pallidus $(+41 \%$ compared to the unlesioned side; Table 1; Fig. 3), dorsolateral caudate $(+10 \%$; Table 1$)$, postcommissural portions of the putamen ( +17 to $+19 \%$; Table 1; Fig. 3), and pedunculopontine nucleus $(+15 \%$; Table 5). Significant decreases in local cerebral metabolism were found in the subthalamic nucleus $(-17 \%$; Table 1; Fig. 4). Other structures in which statistically significant side-to-side differences were found (ventral anterior thalamus, Table 2; parafascicular nucleus, Table 2; premotor cortex, Table 3) showed asymmetries of less than $5 \%$. Because these differences were of such small magnitude, however, it is difficult to attach any functional significance to them.

In none of the 82 structures, with the exception of the nucleus parabrachialis pigmentosus (Table 1), was a statistically significant difference found between the unlesioned side of the hemiparkinsonian monkeys and the corresponding side of the control animals.

These metabolic abnormalities were observed in each animal regardless of the time after MPTP treatment, with the exception of the changes in the putamen, which were not present in the 
Table 1. Rates of glucose utilization ( $\mu \mathrm{mol} / \mathbf{1 0 0} \mathrm{gm} / \mathrm{min})$ in basal ganglia of MPTP-induced hemiparkinsonian monkeys (mean \pm SEM)

\begin{tabular}{|c|c|c|c|c|}
\hline \multirow[b]{2}{*}{ Structure } & \multicolumn{2}{|c|}{ Controls $(n=4)$} & \multicolumn{2}{|c|}{ Hemiparkinsonian $(n=4)$} \\
\hline & Right & Left & Lesioned & Unlesioned \\
\hline Dorsomedial caudate & $53 \pm 4$ & $53 \pm 3$ & $52 \pm 5$ & $53 \pm 4$ \\
\hline Ventral caudate & $45 \pm 2$ & $45 \pm 2$ & $42 \pm 2$ & $44 \pm 2$ \\
\hline Dorsolateral caudate & $49 \pm 2$ & $49 \pm 2$ & $57 \pm 5^{\alpha}$ & $52 \pm 3$ \\
\hline Body of caudate & $57 \pm 3$ & $56 \pm 3$ & $57 \pm 5$ & $53 \pm 5$ \\
\hline Tail of caudate & $52 \pm 4$ & $52 \pm 4$ & $47 \pm 2$ & $48 \pm 3$ \\
\hline Anterior dorsal putamen & $50 \pm 4$ & $50 \pm 4$ & $62 \pm 6$ & $56 \pm 4$ \\
\hline Anterior ventral putamen & $49 \pm 3$ & $50 \pm 4$ & $57 \pm 4$ & $52 \pm 3$ \\
\hline Posterior dorsal putamen & $51 \pm 4$ & $51 \pm 5$ & $63 \pm 5^{b}$ & $53 \pm 4$ \\
\hline Posterior ventral putamen & $56 \pm 4$ & $55 \pm 5$ & $63 \pm 6^{a}$ & $54 \pm 4$ \\
\hline Globus pallidus internal & $29 \pm 1$ & $28 \pm 2$ & $30 \pm 2$ & $28 \pm 2$ \\
\hline Globus pallidus external & $24 \pm 2$ & $25 \pm 2$ & $38 \pm 4^{h, c}$ & $27 \pm 2$ \\
\hline Subthalamic nucleus & $57 \pm 5$ & $58 \pm 5$ & $48 \pm 2^{a}$ & $58 \pm 5$ \\
\hline Claustrum . & $30+1$ & $32 \pm 2$ & $25 \pm 2$ & $26 \pm 2$ \\
\hline Substantia nigra pars compacta & $43 \pm 1$ & $43 \pm 1$ & $30 \pm 1^{b . d}$ & $39 \pm 1$ \\
\hline Substantia nigra pars reticulata & $30 \pm 2$ & $31 \pm 1$ & $28 \pm 3$ & $29 \pm 2$ \\
\hline Ventral tegmental area & $37 \pm 1$ & $36 \pm 1$ & $31 \pm 1^{a, c}$ & $32 \pm 1$ \\
\hline Nucleus paranigralis & $45 \pm 1$ & $45 \pm 1$ & $41 \pm 1^{b}$ & $43 \pm 2$ \\
\hline Nucleus parabrachialis pigmentosus & $40 \pm 1$ & $41 \pm 1$ & $33 \pm 2^{c}$ & $34 \pm 1^{c}$ \\
\hline
\end{tabular}

" $p<0.05$ and $" p<0.01$ side-to-side comparisons (Bonferroni $t$ test following 2-way ANOVA).

" $p<0.05$ and " $p<0.01$ lesioned side vs corresponding side of control (Bonferroni $t$ test following 2-way ANOVA).

" $p<0.05$ unlesioned side vs corresponding side of control (Bonferroni $t$ test following 2-way ANOVA).

monkey studied 1.5 months post-MPTP-infusion. This was the shortest time between intracarotid MPTP infusion and the 2DG procedure. Other changes were present in similar degrees in all animals. Time after MPTP infusion, therefore, did not appear to be a determinant of the distribution of metabolic alterations.

\section{Discussion}

MPTP-induced hemiparkinsonism in monkeys was associated with metabolic changes, ipsilateral to the side of the infusion, in a few specific portions of the basal ganglia and their major projection fields, i.e., SNpc, posterior putamen, external globus pallidus, subthalamic nucleus, pedunculopontine nucleus, ventral anterior thalamus, and premotor cortex. Although the behavioral consequences of destruction of the dopaminergic cells of the SNpc are profound, only a limited number of neural circuits appeared to be affected. These data, therefore, support the view (Wooten and Collins, 1983; Mitchell et al., 1986; Crossman, 1987) that parkinsonism is not a disturbance of the basal ganglia per se, but, more precisely, of selected circuits within this system.

Table 2. Rates of glucose utilization ( $\mu \mathrm{mol} / \mathbf{1 0 0} \mathrm{gm} / \mathrm{min}$ ) in thalamus of MPTP-induced hemiparkinsonian monkeys (mean \pm SEM)

\begin{tabular}{|c|c|c|c|c|}
\hline \multirow[b]{2}{*}{ Structure } & \multicolumn{2}{|c|}{ Comtrols $(n=4)$} & \multicolumn{2}{|c|}{ Hemiparkinsonian $(n=4)$} \\
\hline & Right & Left & Lesioned & Unlesioned \\
\hline Ventral anterior & $38 \pm 2$ & $38 \pm 1$ & $40 \pm 2^{a, b}$ & $39 \pm 2$ \\
\hline Ventral lateral & $42 \pm 1$ & $41 \pm 0$ & $41 \pm 2$ & $41 \pm 2$ \\
\hline Anterior ventral & $57 \pm 3$ & $57 \pm 3$ & $58 \pm 3$ & $58 \pm 3$ \\
\hline Anterior medial & $54 \pm 2$ & $53 \pm 2$ & $50 \pm 1$ & $52 \pm 1$ \\
\hline Centromedian nucleus & $38 \pm 2$ & $39+2$ & $41 \pm 1$ & $41 \pm 1$ \\
\hline Parafascicular nucleus & $51 \pm 3$ & $50 \pm 3$ & $52 \pm 1^{a . b}$ & $50 \pm 1$ \\
\hline Mediodorsal magnocellularis & $56 \pm 4$ & $55 \pm 4$ & $55 \pm 1$ & $55 \pm 1$ \\
\hline Mediodorsal parvicellularis & $55 \pm 2$ & $56 \pm 1$ & $54 \pm 2$ & $55 \pm 1$ \\
\hline Mediodorsal paralamellaris & $57 \pm 2$ & $57 \pm 2$ & $56 \pm 1$ & $60 \pm 2$ \\
\hline Lateral geniculate & $37 \pm 3$ & $38 \pm 3$ & $36 \pm 2$ & $37 \pm 2$ \\
\hline Reticular nucleus & $24 \pm 1$ & $24 \pm 1$ & $24 \pm 2$ & $25 \pm 2$ \\
\hline Ventral posterolateral & $36 \pm 2$ & $36 \pm 1$ & $35 \pm 2$ & $36 \pm 2$ \\
\hline Medial habenula & $32 \pm 3$ & $31 \pm 2$ & $30 \pm 4$ & $30 \pm 3$ \\
\hline Lateral habenula & $50 \pm 5$ & $49 \pm 4$ & $54 \pm 3$ & $51 \pm 4$ \\
\hline Optic tract & $19 \pm 1$ & $20 \pm 2$ & $22 \pm 2$ & $22 \pm 3$ \\
\hline
\end{tabular}

" $p<0.05$ side-to-side comparisons (Bonferroni $t$ test following 2-way ANOVA).

'Although statistically significant, the 2 sides differ by less than $5 \%$. 


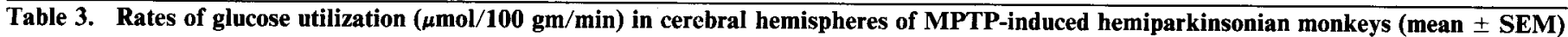

\begin{tabular}{|c|c|c|c|c|}
\hline \multirow[b]{2}{*}{ Structure } & \multicolumn{2}{|c|}{ Controls $(n=4)$} & \multicolumn{2}{|c|}{ Hemiparkinsonian $(n=4)$} \\
\hline & Right & Left & Lesioned & Unlesioned \\
\hline Orbitofrontal cortex & $52 \pm 5$ & $52 \pm 4$ & $53 \pm 2$ & $55 \pm 3$ \\
\hline Dorsolatcral prefrontal cortex & $55 \pm 5$ & $54 \pm 4$ & $55 \pm 4$ & $57 \pm 4$ \\
\hline Anterior cingulate & $39 \pm 2$ & $41 \pm 2$ & $39 \pm 0$ & $40 \pm 1$ \\
\hline Frontal eye fields & $57 \pm 2$ & $58+2$ & $52 \pm 2$ & $54 \pm 2$ \\
\hline Motor cortex & $46 \pm 2$ & $47 \pm 2$ & $42 \pm 2$ & $43 \pm 2$ \\
\hline Premotor cortex & $45 \pm 2$ & $45 \pm 1$ & $43 \pm 1^{a, b}$ & $46 \pm 1$ \\
\hline Striate cortex & $73 \pm 3$ & $73 \pm 2$ & $72 \pm 8$ & $74 \pm 6$ \\
\hline Prestriate cortex & $59 \pm 1$ & $57 \pm 1$ & $59 \pm 8$ & $58 \pm 5$ \\
\hline Entorhinal cortex & $39 \pm 1$ & $39 \pm 1$ & $40 \pm 2$ & $39 \pm 2$ \\
\hline Retrosplenial cortex & $45 \pm 1$ & $45 \pm 3$ & $44 \pm 1$ & $43 \pm 2$ \\
\hline Dentate gyrus & $28 \pm 1$ & $27 \pm 2$ & $29 \pm 2$ & $29 \pm 2$ \\
\hline Hippocampus & $32 \pm 1$ & $33 \pm 1$ & $33 \pm 2$ & $33 \pm 2$ \\
\hline Corpus callosum & $11 \pm 0$ & $10 \pm 1$ & $10 \pm 1$ & $10 \pm 1$ \\
\hline Internal capsule & $11 \pm 2$ & $11 \pm 1$ & $12 \pm 1$ & $12 \pm 1$ \\
\hline
\end{tabular}

${ }^{a} p<0.05$ side-to-side comparisons (Bonferroni $t$ test following 2-way ANOVA).

"Although statistically significant, the 2 sides differ by less than $5 \%$.

\section{Nigrostriatal system}

Ventral mesencephalic tegmentum. The unilateral intracarotid infusion of MPTP was associated with significant reductions in glucosc utilization in the SNpc (Table 1; Figs. 1, 2). The reduction of metabolic activity within the SNpc was not homogeneous; both the mediodorsal portion and the ventral interdigitations extending toward the cerebral peduncle exhibited more pronounced reductions than the laterodorsal aspect of the $\mathrm{SNpc}$ (Fig. 2). Schneider et al. (1987) and German et al. (1988) have reported similar profiles of loss of tyrosine-hydroxylase immunoreactivity in the SNpc of monkeys systemically treated with MPTP. In the SNpc, then, the reductions in glucose utilization are more likely a direct result of cell loss rather than of changes in synaptic or functional activity.

Although damage to the SNpc on the untreated side may occur (cf. Guttman et al., 1988), it is important to note that, first, the unilateral intracarotid administration of MPTP clearly produced the clinical picture of hemiparkinsonism, with little or no behavioral, metabolic, or clinical evidence of impairment on the side contralateral to the untreated hemisphere. Second, dopaminc on the treated side was reduced to less than $90 \%$ of the untreated side. Finally, the damage produced by MPTP was specific to the SNpc with relatively little effect on other dopaminergic systems. MPTP-induced hemiparkinsonism, then, provides a useful model with which to study the effects of dopaminergic denervation of the striatum.

The VTA and the 2 clusters of the tegmental neurons examined in this sludy, nucleus paranigralis and parabrachialis pigmentosus, also showed significant reductions in metabolic rate on the side of the lesion (Table 1). These results are in agreement with the findings of pathological studies of MPTPtreated monkeys (Schneider et al., 1987; German et al., 1988) and parkinsonian patients (Javoy-Agid and Agid, 1980; Uhl et

Table 4. Rates of glucose utilization ( $\mu \mathrm{mol} / 100 \mathrm{gm} / \mathrm{min}$ ) in forebrain and hypothalamus of MPTP-induced hemiparkinsonian monkeys (mean \pm SEM)

\begin{tabular}{|c|c|c|c|c|}
\hline \multirow[b]{2}{*}{ Structure } & \multicolumn{2}{|c|}{ Controls $(n=4)$} & \multicolumn{2}{|c|}{ Hemiparkinsonian $(n=4)$} \\
\hline & Right & Left & Lesioned & Unlesioned \\
\hline Nucleus accumbens & $36 \pm 2$ & $37 \pm 2$ & $34 \pm 1$ & $35 \pm 2$ \\
\hline Olfactory tubercle & $32 \pm 2$ & $33 \pm 2$ & $37 \pm 1$ & $37 \pm 1$ \\
\hline Lateral septum & $27 \pm 1$ & $27 \pm 1$ & $25 \pm 2$ & $25 \pm 2$ \\
\hline Bed nucleus stria terminalis & $23 \pm 0$ & $23 \pm 1$ & $22 \pm 1$ & $22 \pm 1$ \\
\hline Basolateral amygdala & $40 \pm 1$ & $41 \pm 2$ & $36 \pm 3$ & $37 \pm 3$ \\
\hline Lateral amygdala & $36 \pm 2$ & $36 \pm 2$ & $33 \pm 2$ & $35 \pm 2$ \\
\hline Paraventricular hypothalamus & $32 \pm 2$ & $31 \pm 1$ & $30 \pm 1$ & $31 \pm 1$ \\
\hline Lateral hypothalamus & $28 \pm 1$ & $27 \pm 2$ & $26 \pm 1$ & $26 \pm 1$ \\
\hline Substantia innominata & $21 \pm 2$ & $22 \pm 1$ & $20 \pm 1$ & $21 \pm 2$ \\
\hline Mammillary body medialis & $68 \pm 6$ & $68 \pm 6$ & $67 \pm 4$ & $69 \pm 4$ \\
\hline Mammillary body intermediate & $56 \pm 7$ & $56 \pm 6$ & $59 \pm 2$ & $59 \pm 2$ \\
\hline Mammillary body lateralis & $48 \pm 6$ & $47 \pm 6$ & $47 \pm 3$ & $47 \pm 3$ \\
\hline Median eminence & $46 \pm 7$ & $46 \pm 7$ & $41 \pm 4$ & $41 \pm 4$ \\
\hline Anterior commissure & $11 \pm 1$ & $11 \pm 1$ & $11 \pm 1$ & $10 \pm 1$ \\
\hline Anterior white matter & $12 \pm 1$ & $13 \pm 2$ & $11+1$ & $11 \pm 1$ \\
\hline
\end{tabular}




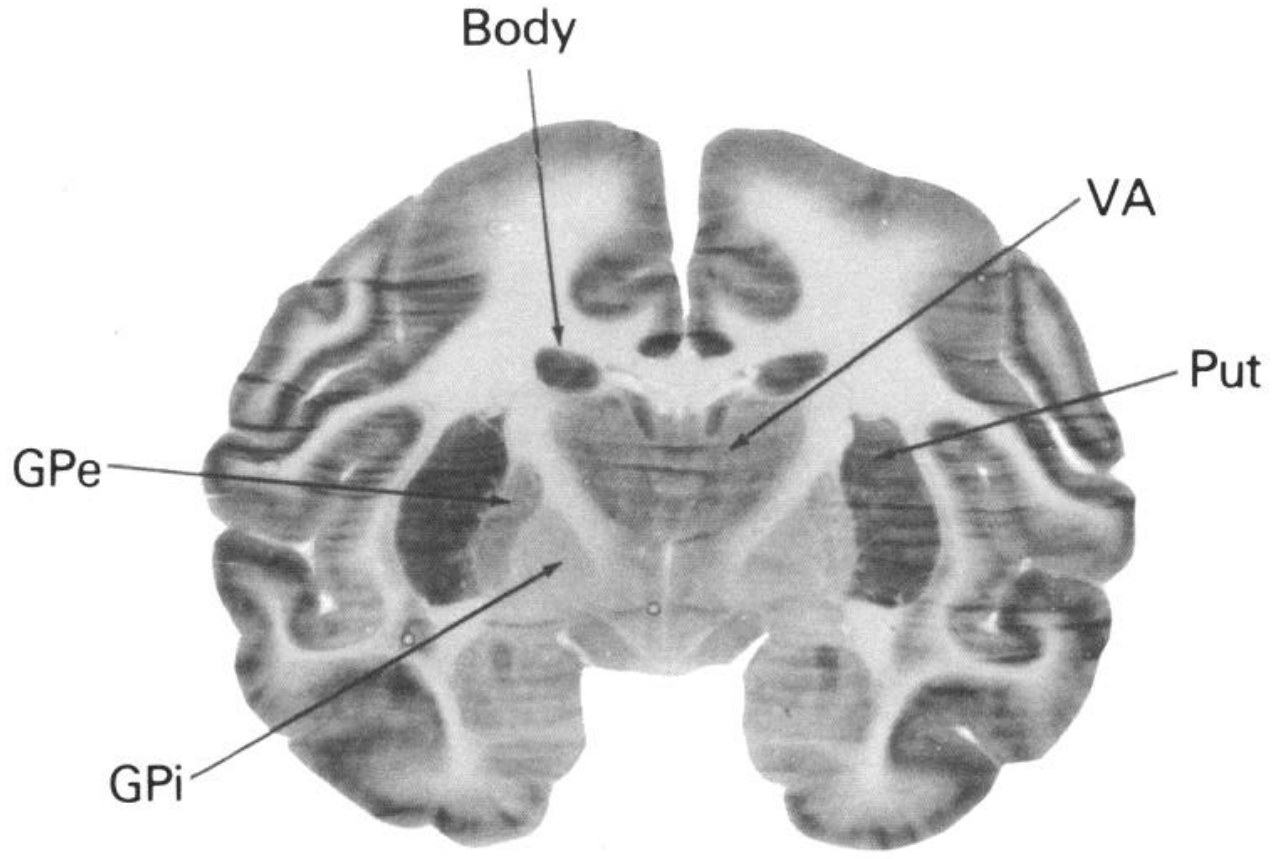

MPTP-Lesioned Side
Figure 3. Autoradiogram of coronal section of brain through the level of the diencephalon. Note the increased labeling in the postcommissural putamen (Put) and external segment of the globus pallidus $(\mathrm{GPe})$ ipsilateral to the lesion of the SNpc. No asymmetry appears in the body of the caudate (Body) or in the internal segment of the globus pallidus $(G P i)$. A slight, yet statistically significant, asymmetry is also evident in the ventrolateral crescent of the ventral anterior thalamus $(V A)$ with increased labeling ipsilateral to the MPTP lesion. The dark horizontal bands crossing the section are technical artifacts that result from folding of the tissue during the processing of the sections. al., 1985). Thus, it appears that degeneration of the VTA is a feature of both experimental parkinsonism and human idiopathic parkinsonism. The reductions in glucose metabolism in the VTA were not accompanied, however, by either neurochemical (Burns et al., 1983; German et al., 1988) or metabolic (Porrino et al., 1987a) changes in its specific mesolimbic and mesocortical target areas (Table 4), unlike lesions of the SNpc in which neurochemical and metabolic changes in target areas of nigral innervation are seen. The only cerebral structure statistically significantly affected bilaterally was the central core of the nucleus parabrachialis pigmentosus. The midline position of this portion of the nucleus suggests that the bilateral effects could be the result of local diffusion of MPTP.

Striatum. Glucose utilization was significantly increased in the posterior putamen and in the most dorsolateral portions of the caudate on the side of the lesion, as compared to the values on the contralateral side. Conversely, no significant metabolic asymmetries were observed in the dorsomedial or ventral caudate, the tail of the caudate, or the more anterior portions of the putamen. The striatal areas in which metabolic hyperactivity was observed coincide with those in which dopamine depletion is most dramatic in human Parkinson's disease (Kish et al., 1988) and in which up-regulation of dopaminergic receptors occurs in hemiparkinsonian monkeys (Joyce et al., 1986; Viola et al., 1988). These data, then, support further the specific importance of the putamen in the symptomatology of parkinsonism (cf. Crutcher and DeLong, 1984).

A possible explanation for this metabolic hyperactivity may lie in the removal of the normally inhibitory influence of dopamine on striatal target neurons (Bloom et al., 1965; Herz and Zieglgaensberger, 1968; Mercuri et al., 1985); the removal of dopaminergic inhibition may result in higher basal activity of striatal interneurons and projection neurons. Furthermore, the reduction of striatal dopaminergic inhibition could result in increased activity of corticostriate excitatory afferents. This striatal metabolic hyperactivity in MPTP-induced hemiparkinsonism, however, is distinct from the findings in MPTP-induced bilateral parkinsonism, in which no metabolic changes were found in the striatum (Porrino et al., 1987a, b). Although the exact mechanisms responsible for this disparity are unclear, these effects may be the result of abnormal activity in any or all of the pathways that have been described through which dopaminergic activity in the nigrostriatal system of one hemisphere can influence neuronal activity on the contralateral side (cf. Cheramy et al., 1979, 1981; Leviel et al., 1979, 1981; Savaki et al., 1983).

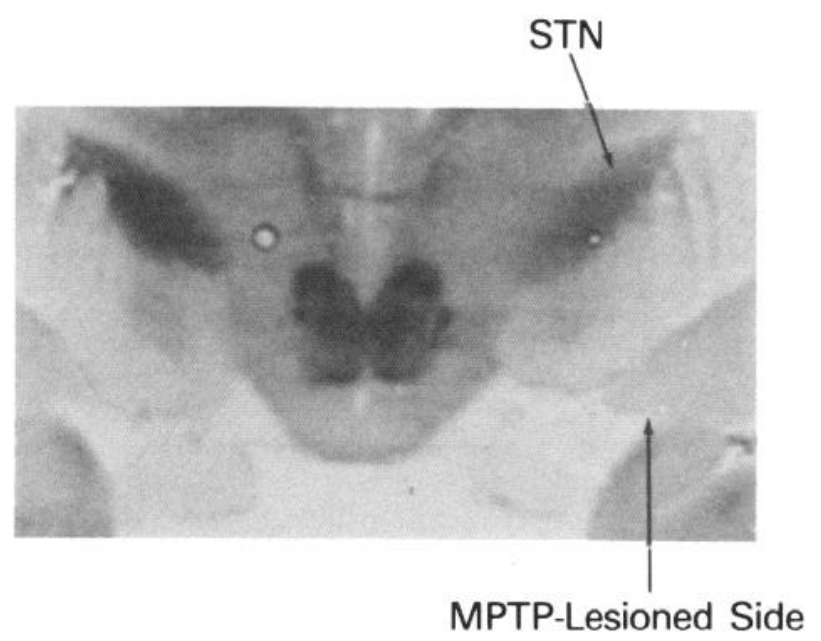

Figure 4. Autoradiogram of portion of coronal section of brain at the level of the subthalamus $(S T N)$. A clear decrease of isotope uptake in the subthalamus is seen ipsilateral to the side with lesion of the SNpc. 
Table 5. Rates of glucose utilization ( $\mu \mathrm{mol} / 100 \mathrm{gm} / \mathrm{min}$ ) in brain stem and cerebellum of MPTP-induced hemiparkinsonian monkeys (mean \pm SEM)

\begin{tabular}{|c|c|c|c|c|}
\hline \multirow[b]{2}{*}{ Structure } & \multicolumn{2}{|c|}{ Controls $(n=4)$} & \multicolumn{2}{|c|}{ Hemiparkinsonian $(n=4)$} \\
\hline & Right & Left & Lesioned & Unlesioned \\
\hline Superior colliculus external & $68 \pm 9$ & $69 \pm 7$ & $68 \pm 8$ & $66 \pm 8$ \\
\hline Superior colliculus internal & $56 \pm 4$ & $54 \pm 2$ & $48 \pm 5$ & $48 \pm 6$ \\
\hline Inferior colliculus & $130 \pm 5$ & $131 \pm 4$ & $124 \pm 3$ & $121 \pm 6$ \\
\hline Red nucleus & $44 \pm 3$ & $44 \pm 3$ & $45 \pm 1$ & $47 \pm 2$ \\
\hline Interpeduncular nucleus & $45 \pm 2$ & $45 \pm 2$ & $41 \pm 1$ & $41 \pm 1$ \\
\hline Pedunculopontine nucleus & $39 \pm 1$ & $39 \pm 1$ & $46 \pm 2^{a}$ & $40 \pm 1$ \\
\hline Locus coeruleus & $37 \pm 2$ & $39 \pm 1$ & $37 \pm 2$ & $37 \pm 2$ \\
\hline Dorsal raphe & $37 \pm 3$ & $37 \pm 3$ & $35 \pm 1$ & $35 \pm 1$ \\
\hline Parabrachialis medialis nucleus & $24 \pm 0$ & $24 \pm 1$ & $22 \pm 0$ & $21 \pm 1$ \\
\hline Parabrachialis lateralis nucleus & $24 \pm 1$ & $24 \pm 1$ & $22 \pm 1$ & $23 \pm 1$ \\
\hline Inferior olive dorsal lamella & $49 \pm 3$ & $50 \pm 3$ & $48 \pm 1$ & $48 \pm 1$ \\
\hline Inferior olive ventral lamella & $48 \pm 3$ & $49 \pm 2$ & $47 \pm 1$ & $47 \pm 1$ \\
\hline Motor dorsal nucleus vagus & $35 \pm 1$ & $34 \pm 1$ & $32 \pm 1$ & $31 \pm 1$ \\
\hline Pyramids & $10 \pm 1$ & $10 \pm 1$ & $8 \pm 1$ & $7 \pm 1$ \\
\hline Cerebellar cortex & $34 \pm 3$ & $35 \pm 2$ & $32 \pm 1$ & $33 \pm 1$ \\
\hline Uvula & $61 \pm 5$ & $59 \pm 5$ & $59 \pm 1$ & $59 \pm 0$ \\
\hline Dentate nculeus & $57 \pm 3$ & $56 \pm 3$ & $53 \pm 2$ & $54 \pm 2$ \\
\hline Interpositus nucleus & $52 \pm 2$ & $50 \pm 2$ & $47 \pm 1$ & $46 \pm 2$ \\
\hline Fastigial nucleus & $46 \pm 2$ & $45 \pm 3$ & $41 \pm 1$ & $40 \pm 1$ \\
\hline Cerebellar white matter & $14 \pm 1$ & $14 \pm 1$ & $14 \pm 0$ & $14 \pm 1$ \\
\hline
\end{tabular}

" $p<0.01$ side-to-side comparisons (Bonferroni $t$ test following 2-way ANOVA).

\section{Striatofugal systems}

Anatomically, the striatum has only 2 main efferent pathways, i.e., to the substantia nigra pars reticulata and to the globus pallidus (Szabo, 1962, 1967; Cowan and Powell, 1966; Fox and Rafols, 1976). The present observations provide further evidence that only the striatopallidal pathway is affected in MPTPinduccd parkinsonism (Wooten and Collins, 1983; Mitchell et al., 1986; Porrino et al., 1987a). The striatonigral pathway was, in fact, metabolically spared with unilateral lesions of the $\mathrm{SNpc}$ (Table 1; Fig. 2), which is consistent with anatomical evidence that in primates the main efferents to the nigra and pallidum originate from separate neuronal populations in the striatum (Feger and Crossman, 1984; Parent et al., 1984; Beckstead and Cruz, 1986).

The external segment of the globus pallidus was metabolically activated (Table 1; Fig. 3) following unilateral MPTP treatment. This alteration, $+40 \%$, was the largest change found in the central nervous system of monkeys following lesions of the SNpc. Elevated rates of glucose utilization in this region are observed as early as $4 \mathrm{~d}$ after systemic MPTP treatment, are maintained several weeks posttreatment (Mitchell et al., 1986; Schwartzman et al., 1988), and are still evident at long posttreatment time periods (Porrino et al., 1987a, b). Similar increases in glucose utilization have also been reported in rats after unilateral 6-hydroxydopamine lesions of the SNpc (Wooten and Collins, 1981 ) and in patients with idiopathic Parkinson's disease studied with the $\left[{ }^{18} \mathrm{~F}\right]$ deoxyglucose method (Martin et al., 1984). These effects are in accordance with the decreases in mean discharge rates of external pallidum neurons following MPTP treatment (Filion et al., 1985; Miller and DeLong, 1987) or clcctrolytic lesions of the SNpc (Filion, 1979). However, the metabolic hyperactivity of the external pallidum is likely to reflect input from the striatum rather than the firing of intrinsic pallidal neurons, because alterations in function-related glucose utilization are a result of changes occurring predominantly in nerve terminals rather than cell bodies (Mata et al., 1980; Kadekaro et al., 1985). Since the projections from the striatum to the pallidum are mainly GABAergic and presumably inhibitory in their effects, an increase in the activity of this circuit, as evidenced by the increased glucose utilization, would result in decreased cell firing, which is seen electrophysiologically (Filion et al., 1985; Miller and DeLong, 1987).

In contrast, no consistent changes in metabolic activity were found in the internal segment of the globus pallidus. These data seem to contradict the results of electrophysiological studies, as well as some previous 2DG studies of parkinsonism. First, single cell recordings of internal pallidum neurons have shown that the mean discharge rate is increased following MPTP treatment (Filion et al., 1985; Miller and DeLong, 1987), and the normal temporal pattern of cell firing becomes disorganized, similar to the changes in the globus pallidus following electrolytic lesion of the SNpc (Filion, 1979). Filion and his colleagues (1985), however, found that average firing rates in the internal pallidum returned to normal several weeks after MPTP treatment although the abnormal temporal firing pattern remained. Second, glucose metabolism in the internal pallidum was increased in 2DG studies conducted $4 \mathrm{~d}$ (Schwartzman and Alexander, 1985) and $21 \mathrm{~d}$ (Mitchell et al., 1986; Schwartzman et al., 1988), but not 3 months (Porrino et al., 1987a), following systemic MPTP administration. It appears, then, that with acute MPTP treatment discharge rates are increased, as in glucose metabolism, but at longer times after MPTP treatment, when rates of glucose use are normal, mean discharge rates are not different from normal. The 2DG method, becausc it integrates over a long time period, is not likely to be able to detect changes in the pattern of firing, but only changes in overall rate.

These data, then, suggest that separate messages can be con- 
veyed from the striatum to each segment of the globus pallidus. Kecent anatomical (Beckstead and Cruz, 1986; Parent et al., 1989) and neurochemical evidence (Haber and Elde, 1981; Beckstead, 1987) indicates that there is scgrcgation of striatal outputs directed to the 2 segments. The present results clearly support the view that not only are striatonigral and striatopallidal fibers functionally segregated, but the portions of the striatopallidal pathway to the internal and external segments of the globus pallidus can be independently activated with a great degree of specificity.

\section{Pallidofugal systems}

The decreases in glucose utilization in the subthalamic nucleus of hemiparkinsonian monkeys are consistent with the changes in metabolism in the external globus pallidus, which is the main source of afferent input to the subthalamic nucleus (Nauta and Mehler, 1966; Kim et al., 1976; Carpenter et al., 1981). Again, because changes in glucose utilization reflect mainly changes in terminal regions rather than in cell bodies, the decreases seen in the subthalamic nucleus are undoubtedly the consequence of the decreased firing rates in the cells of the external segment of the globus pallidus observed following nigrostriatal denervation (Filion et al., 1985; Miller and DeLong, 1987). In addition, interruption of the pathway from the SNpc directly to the subthalamic nucleus (Rinvik et al., 1979), because of the loss of $\mathrm{SNpc}$ neurons, may also contribute to the decrement in glucose utilization in the subthalamic nucleus of hemiparkinsonian monkeys.

Ipsilateral to the lesion of the SNpc, glucose utilization was increased in the pedunculopontine nucleus, which is connected to the hasal ganglia through a massive projection from the internal pallidum (Nauta and Mehler, 1966; Carpenter and Strominger, 1967; Kim et al., 1976) and with the motor system through connections with the premotor cortex (Kuypers and Lawrence, 1967). Alterations in the inputs from these regions are potential sources for this effect. Although the average metabolic rate in the internal segment of the globus pallidus was unchanged in the present study, electrophysiological studies have shown that the temporal firing pattern of internal pallidum neurons is altered after MPTP treatment (Filion et al., 1985; Miller and DeLong, 1987). Such changes in firing pattern may be translated into alterations in the activity of efferents to the pedunculopontine nucleus and, hence, to changes in glucose utilization in this nucleus. Because decreases in metabolic rate in the premotor cortex were also evident, however, it is not possible to identify unequivocally the exact origin of the metabolic alterations in the pedunculopontine nucleus.

The metabolic changes within components of the pallidofugal system are among the most prominent and consistent observed in the present study. The primary projections to and from the pedunculopontine nucleus, like those to and from the subthalamic nucleus, form loops wholly contained within the basal ganglia (cf. Graybiel and Ragsdale, 1979). Such loops may serve as modulators of the overall level of tonic neuronal activity within the extrapyramidal system, thereby setting the "gain" in the basal ganglia. Alterations in this gain setting may be a principal component of the neural basis of parkinsonism.

In addition, there were statistically significant metabolic changes in some thalamic motor nuclei (Table 2), but because the magnitude of these changes was rather small (less than 5\%), it is difficult to be certain of their functional significance. Small metabolic asymmetries, for example, were found in the ventral anterior nucleus, which receives projections from the internal pallidum (Nauta and Mehler, 1966; Kuo and Carpenter, 1973; Kim et al., 1976). If these alterations have any functional significance at all, they, like those in the pedunculopontinc nucleus, may reflect electrophysiological changes in the internal pallidum.

\section{Metabolic mapping of other models of parkinsonism}

There have been a number of 2DG studies of the metabolic consequences of systemic MPTP administration. In some of these investigations (Schwartzman and Alexander, 1985; Mitchell et al., 1986; Palombo et al., 1988; Schwartzman et al., 1988) monkeys were studied at relatively early times when degeneration of the $\mathrm{SNpc}$ was still ongoing, and the rates of glucose utilization in the $\mathrm{SNpc}$ are still high. During approximately the first 4-5 weeks after MPTP administration, perturbations of serotonergic and noradrenergic systems remain, limiting the interpretation of studies carried out during this period (Burns et al., 1983; Chiueh et al., 1984). Hemiparkinsonian monkeys in the present investigation were studied a minimum of 6 weeks after unilateral intracarotid MPTP infusion, so that these local cerebral metabolic abnormalities can appropriately be compared to the metabolic alterations seen in bilateral parkinsonism 2-4 months after the systemic administration of MPTP.

The distribution of metabolic abnormalities in hemiparkinsonism is more complex than in chronic bilateral parkinsonism. While metabolic changes in the SNpc, VTA, external pallidum, and subthalamus are similar in the 2 conditions, hemiparkinsonism is associatcd with metabolic abnormalities in portions of the striatum and thalamus that were not found in bilateral parkinsonism. These differences, as discussed previously, may be the result of the unopposed activity of the nonlesioned hemisphere and its effects on the neural activity in the lesioned hemisphere. The opposite is true for the paralamellar portion of the mediodorsal nucleus of the thalamus and frontal eye field (Porrino et al., 1987a; Ho et al., 1988). Both of these areas, which are involved in the production of voluntary eye movements (for review, see Bruce and Goldberg, 1984), are metabolically abnormal in bilateral parkinsonism, but spared in hemiparkinsonism (Tables 2, 3). Despite the fact that hemiparkinsonian monkeys have deviation of gaze, there does not appear to be a significant decrease in voluntary eye movements as had been seen in bilateral parkinsonism (Ho et al., 1988). This behavioral difference is a possible source of metabolic differences between the 2 models.

In many respects, the present model shares a number of features with the unilateral destruction of the dopaminergic cells by the direct infusion of the neurotoxin 6-hydroxydopamine into the SNpc or the medial forebrain bundle. Studies with the $2 D G$ method of this model in rodents have shown a similar distribution of metabolic alterations in the $\mathrm{SNpc}$ and in the globus pallidus (Wooten and Collins, 1981, 1983). An additional parallel with the present study is the lack of change in deoxyglucose uptake in the hemisphere contralateral to the lesion, and in the entopeduncular nucleus (the homologue of the internal segment of the primate globus pallidus) and the substantia nigra reticulata. One difference is the absence of effects in the subthalamus and the striatum of 6-hydroxydopamine-lesioned rats (Wouten and Collins, 1981, 1983). But because metabolic changes in primate striatum were restricted only to the posterior putamen and dorsolateral caudate, a comparably detailed regional analysis in the rat is necessary for a valid comparison. Increases 
in glucose utilization ipsilateral to the lesion were also noted in the lateral habenula of 6-hydroxydopamine-lesioned rats (Wooten and Collins, 1981), but not in hemiparkinsonian monkeys. This difference between rats and monkeys supports the view that the pallidohabenular connection may be anatomically and functionally different in the two species (Parent and DeBellefeuille, 1983). Another potential explanation is the possible involvement of the ventral tegmental area in 6-hydroxydopamine lesions, not a factor in MPTP-induced parkinsonism. Although the results from metabolic studies of the 2 models are similar in many respects, the MPTP model is more selective, in that the destruction is predominantly of $\mathrm{SNpc}$ rather than both SNpc and VTA dopaminergic cells. Therefore, lesions made with MPTP can reveal more specific information about the unique role of the substantia nigra in the physiology of the basal ganglia and in the production of normal movements.

\section{Behavioral considerations}

The stimulation or lesion of most basal ganglia structures and/ or their output centers is known to elicit turning behavior in experimental animals (for reviews, cf. Dray, 1979; Pycock, 1980). In the present study, however, bilateral asymmetry of metabolic rate in any portion of the caudate or putamen in hemiparkinsonian monkeys was not associated with a greater degree of either spontaneous or apomorphine-elicited turning. There were also no correlations between degree of turning and extent of metabolic asymmetry in other regions implicated as substrates of turning behavior, including the ventroanterior thalamus, intralaminar thalamic nuclei, substantia nigra pars reticulata, and deep layers of superior colliculus. It should be emphasized, however, that the present experiments were performed in chaired, resting animals, which by its very nature limits the possibility of observing asymmetries in behavior to other than deviation of head and gaze. On the other hand, the absence of any behavioral asymmetries indicates that the observed metabolic asymmetries were not secondary to turning behavior, but the direct consequence of the lesion itself, and are more likely related to the other signs of hemiparkinsonism, e.g., bradykinesia, tremor, and rigidity.

The attempt to interpret the present results has been based on sequential processing of information from a primary source of innervation, the $\mathrm{SNpc}$, to a chain of basal ganglia structures. The connectivity of these structures, however, is rather formed by reciprocal innervations that constitute a complex network of many circular loops. This makes it difficult to establish the actual sequence of transynaptic alterations with a method such as the 2DG method, in which time-integrated metabolic maps of the central nervous system are obtained. As complex as the anatomical connections are, however, individual structures within the basal ganglia have preferential connections with only a limited number of targets. Given this, the present data indicate that MPTP-induced hemiparkinsonism in primates is a unilateral nigrostriatopallidal disease associated with transynaptic metabolic changes in the subthalamus, motor thalamus, premotor cortex, and, at a brain-stem level, the pedunculopontine nucleus.

\section{References}

Bankiewicz, K. S., E. H. Oldfield, C. C. Chiueh, J. L. Doppman, D. M. Jacobowitz, and I. J. Kopin (1986) Hemiparkinsonism in monkeys after unilateral internal carotid artery infusion of 1-methyl-4-phenyl1,2,3,6-tetrahydropyridine (MPTP). Life Sci. 39: 7-16.

Beckstead, R. M. (1987) Striatal substance P cell clusters coincide with the high density terminal zones of the discontinuous nigrostriatal dopaminergic projection system in the cat: A study by combined immunohistochemistry and autoradiographic axon-tracing. Neuroscience 20: 557-576.

Beckstead, R. M., and C. J. Cruz (1986) Striatal axons to the globus pallidus, entopeduncular nucleus and substantia nigra come mainly from separate cell populations in cat. Neuroscience 19: 147-158.

Bloom, F. E., E. Costa, and G. C. Salmoiraghi (1965) Anesthesia and responsiveness of individual neurons of the caudate nucleus of the cat to acetylcholine, norepinephrine and dopamine administered by microelectrophoresis. J. Pharmacol. Exp. Ther. 150: 244-252.

Brewer, J. C., J. S. Schneider, T. Hawk, J. M. Hoffman, J. R. Barrio, S. C. Huang, A. Luxen, N. Satyamurthy, J. C. Mazziotta, and M. E. Phelps (1988) Bilateral and hemiparkinsonian monkeys spontaneously recover from the effects of MPTP. Neurosci. Abstr. 14: 389.

Bruce, C. J., and M. E. Goldberg (1984) Physiology of the frontal eye fields. Trends Neurosci. 7: 436-441.

Burns, R. S., C. C. Chiueh, S. P. Markey, M. H. Ebert, D. M. Jacobowitz, and I. J. Kopin (1983) A primate model of parkinsonism: selective destruction of dopaminergic neurons in the pars compacta of the substantia nigra by $N$-methyl-4-phenyl-1,2,3,6-tetrahydropyridine. Proc. Natl. Acad. Sci. USA 80: 4546-4550.

Carpenter, M. B., and N. L. Strominger (1967) Efferent fibers of the subthalamic nucleus in the monkey: A comparison of the efferent projections of the subthalamic nucleus, substantia nigra and globus pallidus. Am. J. Anat. 121: 41-72.

Carpenter, M. B., S. C. Carleton, J. T. Keller, and P. Conte (1981) Connections of the subthalamic nucleus. Brain Res. 224: 1-29.

Cheramy, A., A. Nieoullon, and J. Glowinski (1979) Effects of unilateral nigral application of baclofen on dopamine release in the two caudate nuclei of the cat. Eur. J. Pharmacol. 58: 133-140.

Cheramy, A., V. Leviel, F. Daudet, B. Guibert, M. F. Chesselet, and J. Glowinski (1981) Involvement of the thalamus in the reciprocal regulation of the two nigrostriatal dopaminergic pathways. Neuroscience 6: 2657-2668.

Chiueh, C. C., S. P. Markey, R. S. Burns, J. N. Johannessen, D. M. Jacobowitz, and I. J. Kopin (1984) Neurochemical and behavioral effects of 1-methyl-4-phenyl-1,2,3,6-tetrahydropyridine (MPTP) in rat, guinea pig, and monkey. Psychopharm. Bull. 20: 546-553.

Cowan, W. M., and T. P. S. Powell (1966) Striopallidal projection in the monkey. J. Neurol. Neurosurg. Psychiatry 29: 426-439.

Crossman, A. R. (1987) Primate models of dyskinesia: The experimental approach to the study of basal ganglia-related involuntary movement disorders. Neuroscience 21: 1-40.

Crutcher, M. D., and M. R. DeLong (1984) Single cell studies of the primate putamen. I. Functional organization. Exp. Brain Res. 53: 233-243.

Cummins, A. C., K. S. Bankiewicz, R. J. Plunkett, and E. H. Oldfield (1989) Quantification of the remaining cells in the dopaminergic nuclei of MPTP-treated monkeys: A potential source of sprouting fibers after tissue implants. Presented at the Annual Meeting of the American Association of Neurological Surgeons, Washington, DC.

Dray, A. (1979) The striatum and substantia nigra: A commentary on their relationships. Neuroscience 4: 1407-1439.

Feger, J., and A. R. Crossman (1984) Identification of different subpopulations of neostriatal neurones projecting to globus pallidus or substantia nigra in the monkey: A retrograde fluorescence doublelabelling study. Neurosci. I ett. 49:7-12.

Filion, M. (1979) Effects of interruption of the nigrostriatal pathway and of dopaminergic agents on the spontaneous activity of globus pallidus neurons in the awake monkey. Brain Res. 178: 425-441.

Filion, M., R. Boucher, and P. Bedard (1985) Globus pallidus unit activity in the monkey during the induction of parkinsonism by 1-methyl-4-phenyl-1,2,3,6-tetrahydropyridine (MPTP). Neurosci. Abstr. 11: 1160 .

Fox, C. A., and J. A. Rafols (1976) The striatal afferents in the globus pallidus and in the substantia nigra. In The Basal Ganglia, M. D. Yahr, ed., pp. 237-248, Raven, New York.

German, D. C., M. Dubach, S. Askari, S. G. Speciale, and D. M. Bowden (1988) 1 Methyl-4-phenyl-1,2,3,6-tetrahydropyridine-induced parkinsonian syndrome in Macaca fascicularis: Which midbrain dopaminergic neurons are lost? Neuroscience 24: 161-174.

Goochee, C. W., W. Rasband, and L. Sokoloff (1980) Computerized densitometry and color coding of [14C]deoxyglucose autoradiographs. Ann. Neurol. 7: 359-370. 
Graybiel, A. M., and C. W. Ragsdale (1979) Fiber connections of the basal ganglia. Prog. Brain Res. 51: 239-283.

Guttman, M., H. C. Fibiger, A. Jakubovic, and D. B. Calne (1988) Dose related contralateral dopamine depletion after intracarotid MPTP administration in cynomologous monkeys: Validation of the unilateral MPTP monkey model. Neurosci. Abstr. 14: 9.

Haber, S., and R. Elde (1981) Correlation between met-enkephalin and substance $\mathbf{P}$ immunoreactivity in the primate globus pallidus. Neuroscience 6: 1291-1297.

Herz, A., and W. Zieglgaensberger (1968) The influence of microelectrophoretically applied biogenic amines, cholinomimetics and procaine on synaptic excitation in the corpus striatum. Int. J. Neuropharmacol. 7: 221-230.

Ho, V. W., L. J. Porrino, A. M. Crane, R. S. Burns, I. J. Kopin, and L. Sokoloff (1988) Metabolic mapping of the oculomotor system in MPTP-induced parkinsonian monkeys. Ann. Neurol. 23: 86-89.

Javoy-Agid, F., and Y. Agid (1980) Is the mesocortical dopaminergic system involved in Parkinson's disease? Neurology 30: 1326-1330.

Joyce, J. L., J. F. Marshall, K. S. Bankiewicz, I. J. Kopin, and D. M. Jacobowitz (1986) Hemiparkinsonism in a monkey after unilateral carotid artery infusion of 1-methyl-4-phenyl-1,2,3,6-tetrahydropyridine (MPTP) is associated with regional ipsilateral changes in striatal dopamine D-2 receptor density. Brain Res. 382: 360-364.

Kadekaro, M., A. M. Crane, and L. Sokoloff (1985) Differential effects of electrical stimulation of sciatic nerve on metabolic activity in spinal cord and dorsal root ganglion in the rat. Proc. Natl. Acad. Sci. USA 82: 6010-6013.

Kennedy, C., O. Sakurada, M. Shinohara, J. Jehle, and L. Sokoloff (1978) Local cerebral glucose utilization in the normal conscious macaque monkey. Ann. Neurol. 4: 293-301.

Kim, R., K. Nakano, A. Jayaraman, and M. B. Carpenter (1976) Projections of the globus pallidus and adjacent structures: An autoradiographic study in the monkey. J. Comp. Neurol. 169: 263-290.

Kish, S. J., K. Shannak, and O. Hornykiewicz (1988) Uneven pattern of dopamine loss in the striatum of patients with idiopathic Parkinson's disease. N. Engl. J. Med. 318: 876-880.

Kopin, I. J., A. Zuddas, V. Wiese, R. J. Plunkett, E. H. Oldfield, and K. S. Bankiewicz (1988) Dopamine and tyrosine hydroxylase levels in the cortical and subcortical areas in the MPTP-parkinsonian monkeys. Neurosci. Abstr. 14: 1215.

Kuo, J. S., and M. B. Carpenter (1973) Organization of the pallidothalamic projections in the rhesus monkey. J. Comp. Neurol. 151:201236.

Kuypers, H. G. J. M., and D. G. Lawrence (1967) Cortical projections to the red nucleus and the brain stem in the rhesus monkey. Brain Res. 4: 151-188.

Langston, J. W., P. Ballard, J. W. Tetrud, and I. Irwin (1983) Chronic parkinsonism in humans due to a product of meperidine-analog synthesis. Science 219: 979-980.

Leviel, V., A. Cheramy, and J. Glowinski (1979) Role of dendritic release of dopamine in the reciprocal control of the two nigro-striatal dopaminergic pathways. Nature $280: 236-239$.

Leviel, V., M. F. Chesselet, J. Glowinski, and A. Cheramy (1981) Involvement of the thalamus in the asymmetric effects of unilateral sensory stimuli on the two nigrostriatal dopaminergic pathways in the cat. Brain Res. 223: 257-272.

Martin, W. R. W., J. H. Beckman, C. B. Calne, M. J. Adam, R. Harrop, J. G. Rogers, T. J. Ruth, C. I. Sayre, and D. B. Pate (1984) Cerebral glucose metabolism in Parkinson's disease. Can. J. Neurol. Sci. 11: 169-173.

Mata, M., D. J. Fink, H. Gainer, C. B. Smith, L. Davidsen, H. Savaki, W. J. Schwartz, and L. Sokoloff (1980) Activity-dependent energy metabolism in rat posterior pituitary primarily reflects sodium pump activity. J. Neurochem. 34: 213-215.

Mercuri, N., G. Bernardi, P. Calabresi, A. Cotugno, G. Levi, and P. Stanzione (1985) Dopamine decreases cell excitability in striatal neurons by pre- and post-synaptic mechanisms. Brain Res. 358: 110 121.

Miller, W. C., and M. R. DeLong (1987) Altered tonic activity of neurons in the globus pallidus and subthalamic nucleus in the primate MPTP model of parkinsonism. In The Basal Ganglia II (Advances in Behavioral Biology), Vol. 32, M. B. Carpenter and A. Jayaraman, eds., pp. 415-427, Plenum, New York.

Mitchell, I. J., A. J. Cross, M. A. Sambrook, and A. R. Crossman (1986) Neural mechanisms mediating 1-methyl-4-phenyl-1,2,3,6-tetrahy- dropyridine-induced parkinsonism in the monkey: Relative contributions of the striatopallidal and striatonigral pathways as suggested by 2-deoxyglucose uptake. Neurosci. Lett. 63: 61-65.

Nauta, W. J. H., and W. R. Mehler (1966) Projections from the lentiform nucleus in the monkey. Brain Res. 1: $3-42$.

Palombo, E., L. J. Porrino, K. S. Bankiewicz, A. M. Crane, I. J. Kopin, and L. Sokoloff (1988) Administration of MPTP acutely increases glucose utilization in the substantia nigra of primates. Brain Res. 453: 227-234.

Parent A., and L. DeBellefeuille (1983) The pallidointralaminar and pallidonigral projections in primate as studied by retrograde doublelabeling method. Brain Res. 278: 11-27.

Parent, A., C. Bouchard, and Y. Smith (1984) The striatopallidal and striatonigral projections: Two distinct fiber system in primate. Brain Res. 303: 385-390.

Parent, A., Y. Smith, M. Filion, and D. Dumas (1989) Distinct afferents to internal and external pallidal segments in the squirrel monkey. Neurosci. Lett. 96: 140-144.

Porrino, L. J., R. S. Burns, A. M. Crane, E. Palombo, I. J. Kopin, and L. Sokoloff (1987a) Changes in local cerebral glucose utilization associated with Parkinson's syndrome induced by 1-methyl-4-phenyl1,2,3,6-tetrahydropyridine (MPTP) in the primate. Life Sci. 40:16571664.

Porrino, L. J., R. S. Burns, A. M. Crane, E. Palombo, I. J. Kopin, and L. Sokoloff (1987b) Local cerebral metabolic effects of L-dopa therapy in 1-methyl-4-phenyl-1,2,3,6-tetrahydropyridine-induced parkinsonism in monkeys. Proc. Natl. Acad. Sci. USA 84: 5995-5999.

Pycock, C. J. (1980) Turning behavior in animals. Neuroscience 5. 461-514.

Reivich, M., J. Jehle, L. Sokoloff, and S. S. Kety (1969) Measurements of regional cerebral blood flow with antipyrine-14C in awake cats. J. Appl. Physiol. 27: 269-300.

Rinvik, E., I. Grofova, C. Hammond, J. Feger, and J. M. Deniau (1979) A study of the afferent connections to the subthalamic nucleus in the monkey and the cat using the HRP technique. In Advances in $\mathrm{Neu}$ rology, Vol. 24, L. J. Poirier, T. L. Sourkes, and P. J. Bedard, eds., pp. 53-70, Raven, New York.

Savaki, H. F., M. Deshan, J. Glowinski, and M. J. Besson (1983) Local cerebral glucose consumption in the rat. II. Effects of unilateral substantia nigra stimulation in conscious and halothane-anesthetized animals. J. Comp. Neurol. 213: 46-65.

Schneider, J. S., A. Yuwiler, and C. H. Markham (1987) Selective loss of subpopulations of ventral mesencephalic dopaminergic neurons in the monkey following exposure to MPTP. Brain Res. 411: 144-150.

Schwartzman, R. J., and G. M. Alexander (1985) Changes in the local cerebral metabolic rate for glucose in the 1-methyl-4-phenyl-1,2,3,6tetrahydropyridine (MP'IP) primate model of Parkinson's disease. Brain Res. 358: 137-143.

Schwartzman, R. J., G. M. Alexander, T. N. Ferraro, J. R. Grothusen, and S.S. Stahl (1988) Cerebral metabolism of parkinsonian primates 21 days after MPTP. Exp. Neurol. 102: 307-313.

Sokoloff, L., M. Reivich, C. Kennedy, M. H. DesRosiers, C. S. Patlak, K. S. Pettigrew, O. Sakurada, and M. Shinohara (1977) The [14C]deoxyglucose method for the measurement of local cerebral glucose utilization: Theory, procedure, and normal values in the conscious and anesthetized albino rat. J. Neurochem. 28: 879-916.

Szabo, J. (1962) Topical distribution of the striatal efferent in the monkey. Exp. Neurol. 5: 21-36.

Szabo, J. (1967) The efferent projections of the putamen in the monkey. Exp. Neurol. 19: 463-476.

Uhl, G. R., J. C. Hedreen, and D. L. Price (1985) Parkinson's disease: Loss of neurons from the ventral tegmental area contralateral to therapeutic surgical lesions. Neurology 35: 1215-1218.

Ungerstedt, U. (1971) Stereotaxic mapping of monoamine pathways in the rat brain. Acta Physiol. Scand. 367 (Suppl.): 1-48.

Viola, J. J., L. J. Porrino, K. S. Bankiewicz, A. M. Crane, J. Jehle, and I. J. Kopin (1988) Alteration in the distribution of dopamine D1 and D2 receptors in MPTP-induced hemiparkinsonian monkeys. Neurosci. Abstr. 14: 389.

Wooten, G. F., and R. C. Collins (1981) Metabolic effects of unilateral lesion of the substantia nigra. J. Neurosci. $1: 285-291$.

Wooten, G. F., and R. C. Collins (1983) Effects of dopaminergic stimulation on functional brain metabolism in rats with unilateral substantia nigra lesions. Brain Res. 263: 267-275. 